\title{
Towards Multi-Finger Dexterous Hand Mechanics Control and Tactile Feedback
}

\author{
Ying Liu, Wuhan University of Science and Technology, China \\ (iD) https://orcid.org/0000-0003-2199-0587 \\ Du Jiang, Wuhan University of Science and Technology, China \\ Yibo Liu, Wuhan University of Science and Technology, China \\ Juntong Yun, Wuhan University of Science and Technology, China \\ Dongxu Bai, Wuhan University of Science and Technology, China \\ Gongfa Li, Wuhan University of Science and Technology, China \\ Dalin Zhou, University of Portsmouth, UK
}

\begin{abstract}
The ever-changing demands of industrial automation and space technology have promoted the rapid development of robotics. Traditional robotic end effectors are difficult-to-perform smart operations, so there is an urgent need for a robotic hand to perform complex operations instead of humans. In this article, the authors focus their attention on mechanical control and haptic feedback. Mechanical control and haptic feedback are necessary conditions for the stable and accurate grasping of multi-finger dexterous hands. Tactile perception can provide stiffness and temperature to multi-finger dexterous hands. Important information makes the function of the dexterous hand more perfect. This article introduces the kinematics and dynamics of dexterous hand fingers, as well as the kinematics and dynamics solving equations, then reviews the current sensors and various control driving methods used in dexterous hands, discusses drive control, and compares each method's pros and cons. Finally, the future development of dexterous hands is predicted.
\end{abstract}

\section{KEYWORDS}

Control Drive, Dexterous Hand, Mechanics, Sensory Perception, Tactile Feedback

\section{INTRODUCTION}

Tactile sensation is one of the most complex modes of human perception. The sense of touch comes from a variety of neural arrays, or even the perception of the entire body. The human hand is one of the organs with the most applied haptics(Shang, 2012). The tactile information can reflect the important information as the force between object and finger tip, flatness, which can improve the precise grasping of the end of humanoid robot, thus the humanoid robot can better accomplish the complicated, subtle and dangerous task(Dahiya, 2010)(Ulrika, 2015). The force exerted by the object is explored by the sense of touch and is fed back to the control system by the sense of touch(Peerdeman, 2011). Multi-fingered dexterous hand is characterized by multi-fingered or single-fingered dexterous hand which has multiple degrees of freedom and requires precise control of joint displacement and force, and multi-fingered dexterous hand is characterized by multi-fingered and multi-fingered dexterous 
hand to ensure stability and impedance in grasping process. Most algorithms are specific to specific environments and tasks, and it is difficult to generalize them to new environments and tasks (Zhang, 2017). Therefore, we want to combine tactile feedback with mechanical control to better control the grasping task of multi-finger dexterous hand and improve the generalization. The content of this paper is as follows: Section 2 introduces the mechanics of multi-finger dexterous hand. Section 3 summarizes the robot control drive. Control mainly includes adaptive control, PID control and fuzzy control. Tactile feedback is introduced in section 4. Section 5 summarizes the conclusions.

\section{MULTI-FINGER DEXTEROUS HAND MECHANICS}

The human hand mainly consists of three parts (Zhe, 2016): wrist, palm and finger. The wrist joint is connected to the palm through the (CMC) of the carpometacarpal joint, as shown in Figure 1.

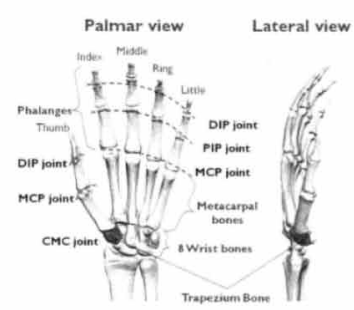

(a) (Zhe, 2016)

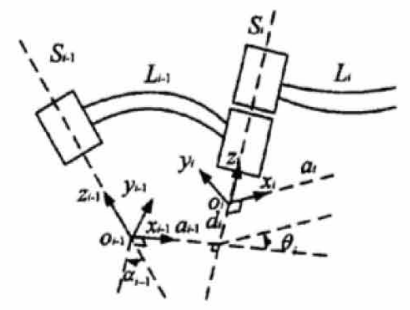

(b) (Mechanics , 2020)

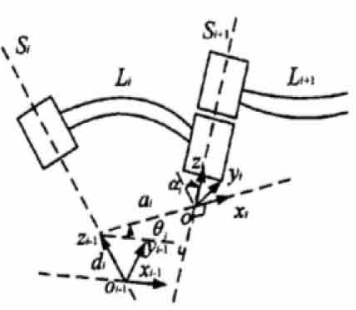

(c) (Mechanics , 2020)

\subsection{Finger Kinematics}

Kinematics is a branch of mechanics which describes and studies the change of object position with time from the geometric point of view. The kinematics is divided into forward solution and inverse solution, and the relative pose relation between the finger linkage is in series form. D-H parameter method is usually used to model forward solutions. Inverse solution is more complex, the commonly used methods are analytical method, iterative method and geometric method.

\subsection{Finger Statics}

Finger statics is the relationship between the force on the tip of the finger and the torque of the joint, when the finger is in force balance. A finger can be approximated by a series of mechanical arms. The Jacobi matrix and determinant can be used to change the speed between the coordinate systems, mapping the relationship to the speed at the end of the finger, the equation of statics can be obtained by taking the differential of the angle of the joint through the Jacobi matrix and determinant equation (Mechanics, 2020).

\subsection{Finger Dynamics}

Dynamics is a branch of theoretical mechanics that deals with the relationship between forces and body motion. Dynamics problems are divided into positive problems and negative problems (Luigi, 2020). Commonly used modeling methods include Newton's Euler method, Joseph-Louis Lagrange 
Table 1. Solving equations of kinematics and dynamics

\begin{tabular}{|l|l|l|}
\hline \multicolumn{1}{|c|}{ Mechanical equation } & \multicolumn{1}{|c|}{ Formula } & \multicolumn{1}{c|}{ Parameter meaning } \\
\hline Positive kinematics & $(x, y, z)=f_{(x, y, z)}\left(\theta_{1}, \theta_{2}, \theta_{3}, \theta_{4}\right)$ & $\theta$ is the joint angle \\
\hline Inverse kinematics & ${ }_{1}{ }_{1} T={ }_{0}^{1} T^{-1} \cdot{ }_{0}^{i} T$ & T is the transformation matrix \\
\hline Statics & $\left.\left[\tau_{i 1}\right]=\left[J_{i j}\right]\right]_{i \leq j}\left[\mathrm{~F}_{(x, y, z)}\right]$ & $\begin{array}{l}\tau \text { is the joint moment vector, F is the } \\
\text { force }\end{array}$ \\
\hline $\begin{array}{l}\text { Newton-Euler } \\
\text { equation(Kinematics) }\end{array}$ & $\begin{array}{l}\vec{F}_{i}=m_{i} \cdot \overrightarrow{\mathrm{a}}_{c i} \\
\vec{f}_{i}=\vec{F}_{i}+{ }^{i} R_{i+1} \vec{f}_{i+1} \vec{I}_{c i} \vec{\varepsilon}_{i}+\vec{W}_{i} \times \vec{I}_{c i} \vec{W}_{i} \\
\vec{n}_{i}=\vec{N}_{i}+{ }^{i} R_{i+1} \vec{n}_{i+1}+\vec{P}_{i} \vec{f}_{i}+\vec{r}_{c i} \vec{F}_{i}\end{array}$ & $\begin{array}{l}W_{i} \text { is angular velocity, } a_{i} \text { is origin } \\
\text { acceleration, } a_{c i} \text { is centroid acceleration }\end{array}$ \\
\hline$\left.\vec{n}_{n+1}=\vec{f}_{n+1}, i=0,1, \cdots n\right)$ & \\
\hline $\begin{array}{l}\text { Lagrange equation } \\
\text { (Kinematics) }\end{array}$ & $\begin{array}{l}L=K-P \\
F_{i}=\frac{\partial}{\partial t}\left(\frac{\partial l}{\partial \dot{X}_{i}}\right)-\frac{\partial l}{\partial X_{i}} \\
T_{i}=\frac{\partial}{\partial t}\left(\frac{\partial l}{\partial \dot{\theta}_{i}}\right)-\frac{\partial l}{\partial \theta_{i}}\end{array}$ & $\begin{array}{l}\text { T is the sum of the external torque of } \\
\text { rotation, and x is the system variable }\end{array}$ \\
\hline
\end{tabular}

method, spin-even number method, Gauss principle and Kane method, and the kinematics and dynamics equations are shown in Table 1.

In view of the problems of large size and complex structure caused by the pursuit of flexibility in traditional dexterous hands. Cai Bo et al. (Cai, 2020) designed a small, compact and highly flexible dexterous hand with three fingers fully driven, and established its three-dimensional model. Using ADAMS software, the dynamics analysis of a single finger is carried out, and the simulation results of ADAMS verify the rationality of the structure design of dexterous hand. Yang Kunming et al. (Yang, 2018) designed a bionic dexterous hand. Based on the Lagrange equation and PD control characteristics, the dynamic model and control scheme of the dexterous hand were solved, and the steering knuckle was independently controlled by PD. The simulation results show that the response characteristics of the single finger tend to be stable. Yang Wucheng et al. (Yang, 2019) designed a mechanical dexterous hand, in view of the dexterous hand job requirement, using Lagrange formula to solve the dynamics equations of dexterous hand, and the use of nonlinear PD controller and adaptive feedback control design, especially the dynamic model of the joints, and the simulation analysis, the results show that the adaptive controller designed by the effect is better. The single-finger control and dynamic analysis of dexterous hands have achieved good results.

\section{DEXTROUS HAND CONTROL DRIVE}

The drive and control of dexterous robot hand is one of the main research directions of dexterous robot hand, and it is an important factor to improve the intelligence level and operation level of dexterous robot hand. Multi-finger driving technology, control technology and grasping theory are the key and difficult problems in the study of anthropomorphic hand. 


\subsection{Dexterous Hand Control}

\subsubsection{Adaptive Control}

Like conventional feedback control, adaptive control is also a control method based on mathematical model (Chun, 2012), as shown in Fig. 2. In general, adaptive control can only solve the uncertainty problem of system model parameters, and it is difficult to ensure the stability under external interference. Therefore, it is necessary to combine adaptive control with other methods to obtain more satisfactory performance.

Figure 2. Adaptive controller

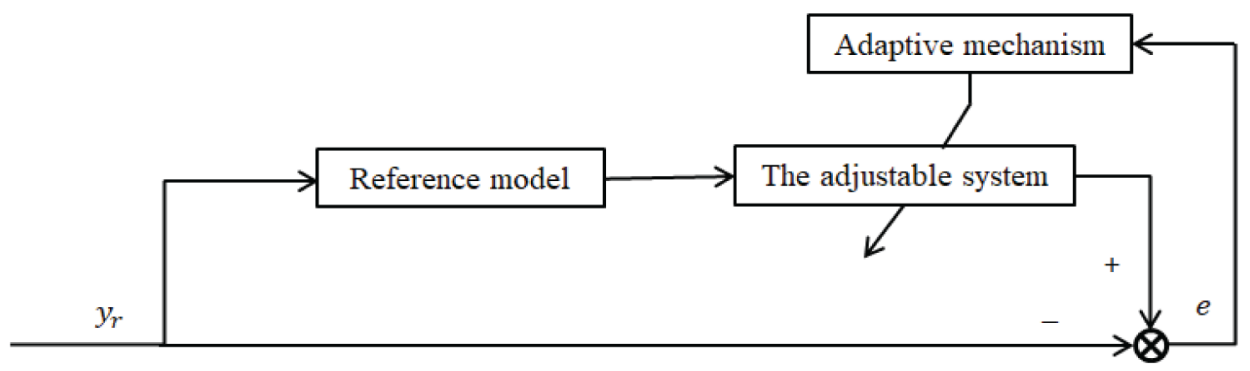

Wang Xingbo (2019) proposed an adaptive impedance controller based on BP neural network to improve the adaptive ability of the contact force system on the premise of ensuring the stability of the system. Experimental results show that the adaptive impedance controller based on BP neural network can reduce the interference.

\subsubsection{PID Control}

The classical PID control method is the most widely used control method. It is simple and reliable. Considering that the input signal is only the position signal of the Angle sensor, it is relatively single, and the signal reading is not continuous and prone to distortion, as shown in Fig. 3.

Taking Titan 4 Seven-function manipulator as an example from Schilling Company, Wang Hongfei et al. (Wang 2020) the mathematical model of electro-hydraulic servo system is established. According

Figure 3. PID Controller

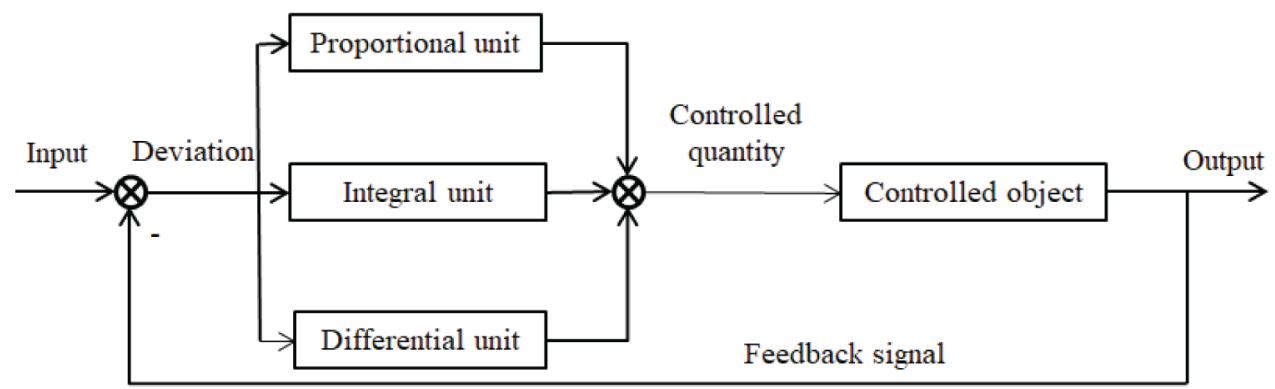


to the working characteristics of the underwater manipulator, PID and feedforward compensation control methods are adopted. The simulation results show that the manipulator has fast response and high control precision. To solve the problem of large tracking error of angular displacement of tandem manipulator, Ye Dan et al. (Ye, 2019) proposed an improved fuzzy PID control method. The improved particle swarm optimization (PSO) fuzzy PID controller is used to control the angular displacement of the manipulator in series. The simulation results show that the tracking error of angular displacement is small.

\subsubsection{Fuzzy Control}

Fuzzy control includes three parts: input fuzzy, fuzzy reasoning, and de-fuzzification. These three links are also called: fuzzifier, fuzzy inference engine, and defuzzifier (Erchao, 2011), as shown in Figure 4.

\section{Figure 4. Fuzzy controller}

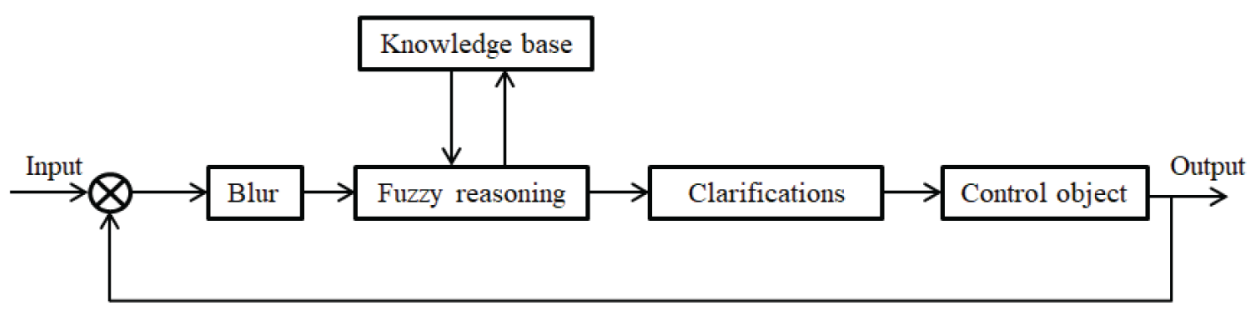

For the trajectory tracking control problem of manipulator with initial error, Yi Xing et al. (Yi, 2020) designed a fuzzy adaptive iterative learning control strategy with initial state learning. The convergence speed and precision are improved obviously, and the effectiveness of the control strategy is verified. In order to improve the accuracy and stability of the motion control of the manipulator of the electric steering gear, Chen Rui et al. (Cheng, 2020) proposed a fuzzy PID intelligent control method based on the orthogonal experimental method. In the traditional PID (proportional, integrated, differentiated, PID) control strategy, the orthogonal experimental design and fuzzy control method are added, the simulation results show that the rise time and adjustment time of the system have a small increase, but the overshoot significantly reduced, improve the accuracy and stability of the system.

The advantages and disadvantages of adaptive control, PID control and fuzzy control are shown in Table 2.

Table 2. Advantages and disadvantages of the three controllers

\begin{tabular}{|l|l|l|}
\hline & \multicolumn{1}{|c|}{ Advantages } & \multicolumn{1}{c|}{ Disadvantages } \\
\hline Adaptive control & $\begin{array}{l}\text { It has simple principle, few parameters and fast } \\
\text { convergence speed }\end{array}$ & $\begin{array}{l}\text { Little prior knowledge and poor } \\
\text { robustness of conventional feedback }\end{array}$ \\
\hline PID control & $\begin{array}{l}\text { Good control effect, no need to establish } \\
\text { mathematical model, good robustness }\end{array}$ & $\begin{array}{l}\text { There is static error, spillover effect } \\
\text { is big }\end{array}$ \\
\hline Fuzzy control & $\begin{array}{l}\text { It is suitable for nonlinear time-varying and large } \\
\text { lag system, and does not need the precise model of } \\
\text { controlled object }\end{array}$ & $\begin{array}{l}\text { Over-dependence on experience and } \\
\text { low steady - state accuracy }\end{array}$ \\
\hline
\end{tabular}


Figure 5. (a) Motor drive; (b)Pneumatic drive; (c) tendon drive

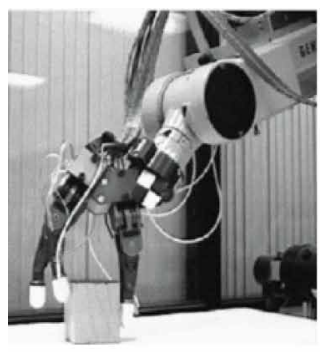

(a) (Mason ,1985)

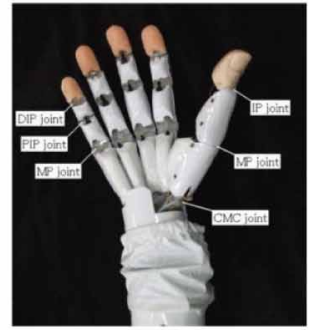

(b) $(\mathrm{Yi}, 2008)$

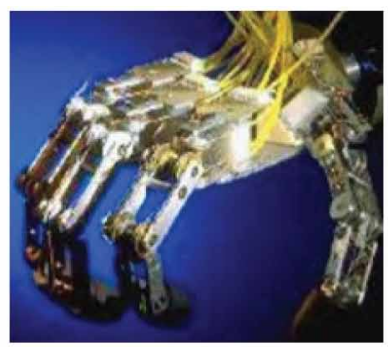

(c) (Liu, 2008)

\subsection{Multi-Finger Dexterous Hand Drive}

One of the core contents of studying smart hand robots is how to drive multi-finger smart hands to achieve multi-modal grasping. Scholars at home and abroad have done a lot of meaningful work in driving methods and structural innovation. In order to achieve characteristics such as multiple degrees of freedom and small size, the requirements of the selected driving method are more stringent. So far, the driving methods of the multi-finger dexterous hand can be roughly divided into: (1) motor driving; (2) fluid driving; (3) other special driving methods.

\subsubsection{Motor Drive}

Motor driven multi-finger dexterous hand can be roughly divided into two types: motor - rope mode and motor - rigid transmission mode. In the early stage, the volume of the micro motor was too large, so the motor was placed outside the hand and the power was transferred through ropes, which could meet the requirements of small size and also provide enough output force.

The DLR-I and DLR II hands (Liu, 2007) were designed and developed by the German Aerospace Center, with a rich sensor system. Each finger is integrated with multiple sensors and is equipped with independent control modules. The dexterous-hand has highly integrated and lightweight design features. DIST hand (Liu, 2008) was developed in Italy. This dexterous hand was driven by tendon to realize movement control, which has the advantages of small size and light weight, as shown in Fig.5(c). The Stanford/JPL smart hand developed by Salisbury (Xiao, 2001) (Mason, 1985) is equipped with a variety of sensors in the motor position, which is also driven by the motor and rope to achieve precise grasping, as shown in Fig.5(a). With the emergence of micro stepping motor with small volume and large torque, rigid drive is adopted to make up for the shortage of rope drive. Robonuat hand (Lovchik, 1999) (Difler, 2011) is driven by brushless DC motor. The whole hand contains a variety of sensors, such as torque sensor, joint position sensor, pressure sensor, etc. Motor driven multi-finger dexterity hand technology is relatively mature, fast response, good stability, but there are still the following deficiencies: (1). Low control accuracy. (2). Lack of flexibility in grasping. (3). Poor stability of rope transmission.

\subsubsection{Fluid Drive}

Fluid transmission is the use of liquid, gas as the conduction medium. Hydraulic transmission is the transmission mode of energy transfer through liquid medium. Pneumatic drive method is to use gas to make the dexterous hand cavity contraction and expansion, in order to achieve the purpose of movement. The channel expands and deforms when air pressure is applied. However, due to its flexibility, its motion trajectory is difficult to control and its efficiency is relatively low, as shown in Fig. 5 (b). The C5 (Yi, 2008) dexterous hand designed by Shadow (Mouri, 2011) uses 40 artificial 
muscles to drive the movement of each joint through ropes and pulleys. It combines air pressure and fingertip torque to accomplish almost all movements of the human hand. Yuki Honda et al. (Honda, 2010) developed a five-fingered dexterous hand with low pressure drive and pneumatic control, and carried out bionic design on the finger structure.

The new pneumatic multi-finger dexterous hand is directly driven by FPA. It is simple in structure, easy to control and has good passive flexibility. Although the pneumatic transmission device has the advantages of light weight, low cost and easy operation, it also has the following disadvantages: (1). The working efficiency of pneumatic transmission device is high, The control system is more complex when there are too many drives. (2). Poor load and impedance capacity, not easy to model and control. Hydraulic motor can provide greater output force, but due to leakage and liquid compression, resulting in hydraulic drive efficiency is not high, the transmission ratio is not accurate, not easy to maintain, large volume and easy to pollution.

\subsubsection{Other Special Drives}

This kind of drive includes shape memory alloy drive (SMA), magnetostrictive drive, electrostatic drive and so on. SMA is filamentous or flaky generally, more embedded in the material interior, through SMA heating, can produce stress and strain.

To solve the problem of small displacement of control drive, Li Dahong et al.(Li,2019) proposed a new SMA driver based on resistance feedback control, and developed a three-fingered Dexteric hand-rope driven actuator. A three - fingered dexterous hand is designed with modular idea. Three fingers have 8 degrees of freedom in total, and the grasping ability of three dexterous hands is verified by experiments. The results show that the output of SMA driver can reach more than $8 \%$ of the total drive length. Modular underactuated finger structure design is adopted, directly using shape memory alloy wire as the drive and drive components. Hao Lina et al. (Hao, 2017) designed a drive displacement amplification structure, and used permanent magnets as the reset structure to realize the integrated lightweight design of drive and drive.

\section{TACTILE FEEDBACK}

The sense of touch is one of the important non-visual information sources. Tactile feedback refers to the feedback of information to the control part of the dexterous hand by means of a sensor or sensor system, which facilitates better interaction with the outside world.

\subsection{Smart Hand Sensor}

Multi-finger dexterous hand in interaction with the outside world, their own sensor is very important, so various sensors are integrated into the dexterous hand ontology, such as torque sensor, joint position sensor, temperature sensor, tension sensor, slide tactile sensor on the surface of the sensor, fingers, and even some depth cameras are integrated into a dexterous hand. Photoelectric sensors and piezoresistive sensors are the main tactile sensors in dexterous hands.

\subsubsection{Photoelectric Sensors}

Photoelectric sensor produces photoelectric effect after receiving visible light irradiation, converts photoelectric signal into electrical signal output, uses the transmission and reflection of light, can measure the size, displacement, speed and other physical quantities.

Konstantinova et al. (Konstantinov, 2017) proposed an integrated fingertip force sensor design that uses a fiber-based approach to adjust light intensity through force. It consists of four sensing elements that form a tactile array that is integrated with the fingertip and can sense the light contact that would normally occur during the initial grip. Li Tao et al. (Li, 2018) described a tactile sensor using PVDF piezoelectric film as the sensing material. The output pressure signals of PVDF are converted by signal conditioning circuit, and the different characteristics of tactile and slip signals 
are analyzed. The test results show that tactile signals and sliding signals can be analyzed effectively in three dimensional space.

\subsubsection{Piezoresistive Sensors}

Piezoresistive sensor is a sensor made by using piezoresistive effect of semiconductor materials and integrated circuit technology. Piezoresistive sensors can measure pressure, tension, differential pressure and other physical quantities.

In order to realize the positioning and tactile force perception of grasping targets by underwater dextrous hands in deep water environment, Zhang Jianjun et al. (Zhang, 2018) designed an array tactile force measurement sensor, and analyzed the analytical solutions of deformation and stress at the bottom of the cube cup using elastic mechanics and plate and shell theory. Zhang Yangyang et al. (Zhang 2,019) proposed a calculation method for fingertip motion trajectory and a pose motion capture algorithm, and established the fingertip motion space master-follower hand contour. Experimental results show that the proposed gesture motion capture algorithm can obtain the spatial contour of fingertip motion of master and slave hands, and the calculation error of fingertip motion trajectory is controlled within $2.8 \mathrm{~mm}$.

In intelligent sliding problem of robot hand grasping objects, Zhang Zi Chao Deng (Zhang, 2020) designed a kind of high sensitive magnetostrictive tactile sensor, according to the inverse magnetostrictive effect, euler Bernoulli beam structure mechanics principle and the hook theorem, the establishment of a tactile sensor friction test model, and by using the sensor for sliding detection, sets the threshold value to 0.05 . The experimental results show that when the bias magnetic field is $4.2 \mathrm{kA} / \mathrm{m}$ and the friction force is $4 \mathrm{~N}$, the peak output voltage of the sensor is $256 \mathrm{mV}$ and the sensitivity is $64 \mathrm{mV} / \mathrm{N}$.

\subsection{Sensor Systems}

In order to achieve stable and precise grasping of smart hands, the sensor system is the key to intelligent control of smart hands to achieve smart operation. Sensors equipped with multi-finger dexterous hands are generally divided into the following three categories (Maekawa, 1996):

1. Gripper attitude sensor: It mainly includes joint Angle sensor, joint torque sensor and fingertip force sensor.

2. Grasp state sensor: It mainly refers to the tactile sensor to obtain the contact state between fingers and objects. It can sense the contact position between fingers and objects in real time, so as to avoid unnecessary grasping, such as grasping at the edges and corners of objects, etc., and it can also sense the sliding trend of objects.

\section{CONCLUSION}

In the past, dexterous hand control and perception have been important factors affecting its stability and accurate grasp. Nowadays, due to the rapid development of sensor technology, the research on the sensing ability of smart hands is also gradually deepening. In this paper, the sensor and control driver of the intelligent hand are summarized, and the kinematics and dynamics of the intelligent hand are emphatically discussed, which can play a role in this field. As more people explore the human hand and more researchers delve into it, dexterous hand research is predictably on a new plateau. They put forward their development expectations from three aspects:

1. Mechanics: The data calculated in theory will be different from the actual control process, which will affect the stable grasp of multifingered dexterous hand.As a result, it is expected to 
be able to study the hand grasping a heavy object, which will rotate in one direction to balance the weight of the object.

2. Control algorithm: Multi-finger coordination control, more accurate grasp motion planning and force planning, to achieve a more stable grasp, but at present a single control method is difficult to achieve good results, it is better to combine a variety of control methods.

3. Tactile feedback: For fingertip materials, the requirements of multi-finger intelligent manipulator are also very high. The sensor system can give back information at the same time, such as the temperature, hardness, size and roughness of an object, just as the human hand can sense the temperature, hardness, size and roughness of an object. Therefore, it is expected that sensory feedback can be applied to machining accuracy.

\section{ACKNOWLEDGMENT}

This work was supported by grants of the National Natural Science Foundation of China (Grant Nos.52075530,51575407, 51505349, 61733011, 41906177,52075530); the Grants of Hubei Provincial Department of Education (D20191105); the Grants of National Defense Pre-Research Foundation of Wuhan University of Science and Technology (GF201705) and Open Fund of the Key Laboratory for Metallurgical Equipment and Control of Ministry of Education in Wuhan University of Science and Technology (2018B07,2019B13) and Open Fund of Hubei Key Laboratory of Hydroelectric Machinery Design \& Maintenance in Three Gorges University(2020KJX02). 


\section{REFERENCES}

Archer, J. R., \& Blenkinsop, P.T. (1996). Actuation for industrial robots. Archive Proceedings of the Institution of Mechanical Engineers, (200), 85-89.

Cai, B., Gao, L. H., \& Song, X. G. (2020). Structure design and contact force planning of three-finger dexterous hand. Mechanical Design and Manufacturing.

Chunyun, P., \& Liji, G. (2012). General industrial robot motion simulation system IRKSS. Robots, 49(2), 94-97.

Craig, J. J. (1987). Introduction to robotics: Mechanics and control. Automatica, 23(2), 263-264.

Dahiya, R. S., Metta, G., \& Valle, M. (2010). Tactile sensing: From humans to humanoids. IEEE Transactions on Robotics, 26(1), 1-20.

Difler, M. A., Mehling, J., \& Abdallah, M. E. (2011). Robonaut 2 - The first humanoid robot in space. In International Conference on Robotics and Automation. IEEE.

Erchao, L. (2011). Research on robot force control technology in unascertained environment. Lanzhou University of Technology.

Hao, L.N., Guo, S.F., \& Cheng, Y. (2017). Design and research of human-like finger transmission structure based on SMA wire drive. Mechanical Transmission, (2), 104-107.

Honda, Y., Nishikawa, A., \& Miyazaki, F. (2010). 2P1-D27 Control of pneumatic five-fingered robot hand using antagonistic muscle ratio and antagonistic muscle activity: Joint angle control of one degree-of-freedom finger model. Institute of Electrical Research, (1), 337-342.

Konstantinov, J., Stilli, A., \& Althoefer, K. (2017). Fingertip fiber optical tactile array with two-level spring structure. Sensors (Basel), 17(10), 2337.

Li, H. D., Jin, Y. Z., \& Guo, Z. W. (2019). New shape memory alloy driver with three finger dexterous hand design. Journal of Engineering Design, 5.

Li, T., Xie, D., \& Wu, H. (2018). Design of tactile sensor of humanoid manipulator. Journal of Changzhou Institute of Technology, 31(5), 22-25.

Liu, H., Meusel, P., \& Seitz, N. (2007). The modular multisensory DLR -HIT-Hand. Mechanism and Machine Theory, 42(5), 612-625.

Liu, H., Wu, K., \& Meusel, P. (2008). Multisensory five-finger dexterous hand: The DLR/HIT Hand I. Proc IEEE/RSJ Int Conference on Intelligent Robots \& Systems, 3692-3697.

Lovchik, C. S., \& Diftler, M. A. (1999). The Robonaut hand: a dexterous robot hand for space. In IEEE International Conference on Robotics \& Automation. IEEE.

Lu, L. H., \& Liu, Q. (2015). The design and implementation of a manipulator for five degree of freedom laparoscopic virtual surgery. 2015 China Biomedical Engineering Joint Academic Annual Conference.

Luigi, R., Aleksandr, S., Salvatore, S., \& Francesco, T. (2020). A hybrid tyre model for in-plane dynamics. Vehicle System Dynamics, 58(7).

Maekawa, H., Tanie, K., \& Komoriya, K. (1996). Dynamic grasping force control using tactile feedback for grasp of multifingered hand. Proceedings - IEEE International Conference on Robotics and Automation, 2462-2469.

Mouri, T., Endo, T., \& Kawasaki, H. (2011). Review of Gifu Hand and its application. Mechanics Based Design of Structures and Machines, 39(2), 210-228.

Peerdeman, B., Boere, D., \& Witteveen, H. (2011). Myoelectric forearm prostheses: State of the art from a usercentered perspective. Journal of Rehabilitation Research and Development, 48(6), 719-737.

Shang, W., \& Cong, S. (2012). Nonlinear computed torque control for a high-speed planar parallel manipulator. Mechatronics, 19(6), 987-992.

Ulrika, W., \& Ingela, C. (2015). Forearm amputees' views of prosthesis use and sensory feedback. J Hand Therap Off J Am Soc Hand Therap, 28(3), 269. 
Wang, H.F., Yan, Y., \& Luo, L.B. (2020). Research on feed-forward compensation control strategy of hydraulic servo system of underwater robot. Control and Information Technology, (3), 8-13.

Wangle, X. B., \& Ge, S. (2019). Adaptive impedance control of dexterous hand driven by tendon based on BP neural network. Information Technology and Network Security, 38(4), 71-75.

Yang, K. M., Shen, J. H., \& Ma, X. Y. (2018). Finger design and control of bionic dexterous hand. Coal Mine Machinery, 363(5), 34-36.

Yang, W. C., Ma, X. Y., \& Song, W. X. (2019). Research on design and control of mechanical dexterous hand. Automation Technology and Application, 38(6), 93-97.

Yang, W. C., Ma, X. Y., \& Song, W. X. (2019). Research on design and control of mechanical dexterous hand. Automation Technology and Application.

Ye, D., \& Wang, D. L. (2019). Research on tracking error of tandem manipulator using improved fuzzy PID control. Journal of Jinggangshan University, 125(03), 79-82.

Yi, X., Cheng, J., \& Liao, X. D. (2020). Research on trajectory tracking control of manipulator based on adaptive iterative learning. Electric Drive, 50(3), 45-50.

Yijun, L., \& Yiwei, L. (2008). Research on finger and impedance control of HIT robot dexterous hand. Machinery Manufacturing, (7), 5-8.

Zhang, J. J., Liu, W. D., \& Zhang, Y. W. (2018). Tactile force measurement sensor for underwater dexterous hand based on mems. Journal of Shanghai Jiaotong University, 52(1), 76-82.

Zhang, S., Li, X., \& Zong, M. (2017). Learning k for knn classification. ACM Transactions 01"1 Intelligent Systems and Technology (TIST), 8(3), 43.

Zhang, Y. Y., Huang, Y., \& Liu, J. X. (2019). Sensor design for gesture motion capture and master-slave hand motion mapping. Robot, 41(2), 156-164.

Zhang, Z. C., Wang, B. W., \& Qi, S. W. (2020). Design and Research of a magnetostrictive tactile sensor for manipulator stable grasping. Mechanical and Electrical Engineering, 300(2), 110-114.

Ying Liu received his BS degree in Coll Huanggang Normal University, Huanggang, China. He is currently occupied in his MS degree in Wuhan University of Science and Technology, Wuhan. His currently interests biological manipulator and image processing.

Du Jiang was born in Hubei province, P. R. China, in 1993. He received B.S. degree in mechanical engineering and automation from Wuhan University of Science and Technology, Wu Han, China, in 2017. He is currently occupied in his M.S. degree in mechanical design and theory at Wuhan University of Science and Technology. His current research interests include image processing and intelligent controls.

Yibo Liu graduated from Henan University of Science and Technology and is currently studying for a master's degree in Wuhan University of Science and Technology. His main research direction is dexterous hand and EMG signal.

Juntong Yun received his BS degree in Wuhan University of Science and Technology, Wuhan, China. He is currently occupied in his MS degree in Wuhan University of Science and Technology, Wuhan. His currently interests include intelligent robot and image processing.

Dalin Zhou is currently a Senior Lecturer in Computer Science with the School of Computing, the University of Portsmouth, UK. His current research interests include signal processing, machine learning, computational intelligence, wearable sensing and analysis, assistive robotics, rehabilitation intelligence, human behaviour analysis and multimodal sensor fusion. His current research contributes to the monitoring and rehabilitation of human motor function, improving the daily life activity and working capability for both the disadvantaged group of limb-impaired patients and the aging community. As an early career researcher, he has published more than 30 refereed journal and conference papers in his interested research area. He has led the research projects funded by EU Commission, European Regional Development Fund as the Principal Investigator and Co-Investigator. He has served as a lead guest editor for International Journal of Humanoid Robotics (IF:1.394) and Sensors (IF:3.275). 